\title{
Epidemiology in Mixed Host Populations
}

\author{
K. A. Garrett and C. C. Mundt
}

First and second authors: Department of Botany and Plant Pathology, 2082 Cordley Hall, Oregon State University, Corvallis 97331-2902; first author: Centro Internacional de la Papa, Apartado 1558, Lima 12, Peru.

Current address of K. A. Garrett: Department of Plant Pathology, Kansas State University, Manhattan 66506-5502.

Accepted for publication 27 July 1999.

\begin{abstract}
Garrett, K. A., and Mundt, C. C. 1999. Epidemiology in mixed host populations. Phytopathology 89:984-990.

Although plant disease epidemiology has focused on populations in which all host plants have the same genotype, mixtures of host genotypes are more typical of natural populations and offer promising options for deployment of resistance genes in agriculture. In this review, we discuss Leonard's classic model of the effects of host genotype diversity on disease and its predictions of disease level based on the proportion of susceptible host tissue. As a refinement to Leonard's model, the spatial structure of host and pathogen population can be taken into account by

be taken into account by considering differences in race-specific resistance among host genotypes, compensation, plant competition, and competitive interactions among pathogen genotypes. The magnitude of hostdiversity effects for particular host-pathogen systems can be predicted by considering how the inherent characteristics of a system causes it to differ from the assumptions of the classic model. Because of the limited number of studies comparing host-diversity effects in different systems, it is difficult at this point to make more than qualitative predictions. Environmental conditions and management decisions also influence hostdiversity effects on disease through their effect on factors such as host density and epidemic length and intensity.
\end{abstract} considering factors such as autoinfection, interaction between host size and pathogen dispersal gradients, lesion expansion, and host carrying capacity for disease. The genetic composition of the host population also can
Additional keywords: cultivar mixtures, genetic diversity, variety mixtures.

notype mixtures of agricultural crops is an attempt to reduce the selection pressure for pathogens that can overcome valuable forms of disease resistance in crop plants (5). Second, selection pressures from disease may shift the composition of host populations over time. Our discussion, however, will focus on the role of epidemiological factors in host-diversity effects, emphasizing genotype mixtures within a single plant species infected with a single pathogen species.

\section{THE CLASSIC MODEL OF HOST-DIVERSITY EFFECTS} diversity within a host plant population. Our goal is to put current studies of host mixtures in a context that allows prediction of which host-pathogen systems are likely to be affected by host diversity and how environment and management methods influence these effects. Knowledge of the role of some epidemiological factors is lacking, and we will discuss areas that need greater attention.

We define a host-diversity effect in an epidemiological context in terms of the disease level (severity or incidence) in a mixture of host genotypes compared with the mean disease level in singlegenotype populations of each of the host genotypes. The levels in single-genotype populations are appropriately weighted depending on the proportion of the mixture comprised by each genotype. If there is a host-diversity effect, it will tend toward reduced disease in mixtures compared with single-genotype populations, but increased disease is predicted in some circumstances.

Two important factors in determining the long-term effects of host diversity on disease will not be addressed in detail in this review. First, pathogen evolution will be affected. One motive for using ge-

Corresponding author: K. A. Garrett; E-mail address: kgarrett@plantpath.ksu.edu

Publication no. P-1999-0831-01V

(c) 1999 The American Phytopathological Society

\section{Simplest Mixtures}

Leonard's classic model of host-diversity effects on disease (35) is based on a single pathogen genotype in simple mixtures of one susceptible and one immune plant genotype. The spatial configuration of host genotypes and pattern of inoculum dispersal are not considered. This spatial simplification may be conceptualized as the assumption that the two plant genotypes are completely mixed in space or that any inoculum produced is randomly distributed throughout the field. This simple model is relevant both to mixtures of two species in which only one species is a host to the same pathogen and to mixtures of two genotypes within a species with different race-specific resistance-one component being immune to all local races. We will refer to such systems as simplest mixtures, because they represent the most simplified system in which the effects of host diversity can be studied. In empirical studies, pairs of a susceptible and an immune or highly resistant host genotype offer a streamlined system for studying whether there is likely to be a host-diversity effect in more complicated mixtures of a host species. These simple mixtures may be of economic interest in their own right when susceptible host genotypes have superior agronomic characteristics that merit protection through deployment in combination with an agronomically inferior, but resistant, genotype. 


\section{Leonard's Predictions}

For simplest mixtures, Leonard (35) predicted that the reduction in disease due to decreased susceptible host tissue would follow

$$
x^{\prime} / x_{o}=m^{n} x / x_{o}
$$

where $x$ is the proportion of infected host tissue in a population composed only of the susceptible genotype, $x^{\prime}$ is the proportion of infected host tissue in the mixture, $x_{o}$ is the proportion of host tissue initially infected, $m$ is the proportion of susceptible plants in the host mixture, and $n$ is the number of generations of disease increase (i.e., the proportion of infected host tissue for the susceptible genotype in simplest mixtures will be $m^{n}$ times the proportion in a population composed of only the susceptible genotype, potentially a very substantial reduction in disease severity). For example, there should be only one-eighth as much disease on susceptible plants in a $50 \%$ susceptible mixture after only three generations of pathogen increase from the primary generation. This model predicts that disease severity will decrease logarithmically as resistant plants are added to a mixture (a diminishing return to resistance). This prediction has been corroborated empirically (reviewed in Mundt and Browning [45]). The model also predicts that host-diversity effects for reduced disease will be greater as more pathogen generations elapse during an epidemic, whether through shorter pathogen generation times or lengthier epidemics.

The results illustrate a fundamental mechanism for reduction in disease due to a reduction in the proportion of susceptible tissue. The way in which real host-pathogen systems differ from simplest mixtures determines to what degree real systems experience the same effect.

\section{SPATIAL REFINEMENTS TO THE CLASSIC MODEL}

One of the assumptions of simplest mixtures is that host tissues and pathogen inoculum are completely mixed in space. The assumption does not hold for real systems because of inherent patterning of host genotypes and disease. Although a susceptible host plant will be influenced by the overall population effect on inoculum load, as modeled by Leonard (35), it may be most influenced by inoculum produced on its own tissues and neighboring plants (22).

\section{Autoinfection}

Autoinfection (sensu Robinson [59]) is the proportion of pathogen inoculum retained on the same host plant on which it was produced. For asexually reproducing pathogens, inoculum produced on a given host plant will be virulent on that plant, aside from the potential effects of induced resistance. The degree of autoinfection is determined by the interaction between the pathogen's dispersal gradient and the size of a single host plant. When plant size is large relative to the spatial extent of propagule dispersion, a high percentage of infection will be autoinfection (Fig. 1A); when plant size is small relative to propagule dispersion, a lower percentage of infection will be autoinfection (Fig. 1B).

Steeper dispersal gradients have been predicted to result in smaller host-diversity effects on disease $(20,49)$. In the field, splash-dispersed pathogens often provide smaller host-diversity effects than do wind-dispersed pathogens $(1,12,25,31,37,44,46,52,53)$, most likely due to the steeper dispersal gradients of splash-dispersed pathogens (19). Wind-dispersed pathogens may produce a large host-diversity effect for reduced disease because propagules tend to be more evenly mixed throughout host plants. The effect may be lost, however, if propagules are so easily dispersed and abundant that high levels of outside inoculum flood a field. Soilborne pathogens have been studied less, and because of much slower rates of dispersal, it may be assumed that the host-diversity effect will be smaller for soilborne than for aerially dispersed pathogens. However, VilichMeller (61) found large reductions in stem rot diseases in mixtures of small-grain species. Little is known about host-diversity effects on virus and insect-vectored diseases, for which insect behavior in a mixed host population would come into play. Power (58) found variable effects of oat cultivar mixtures on aphids transmitting barley yellow dwarf virus, but there was generally reduced virus infection in mixtures.

Conceptualization of spatial patterning of the host can be refined by considering the genotype unit area (GUA): the area occupied by an independent unit of host tissue of the same genotype (45). The GUA often may be the size of a single plant, but it may be smaller if host tissues are intertwined in the field or larger for clonally reproducing plants or agricultural plants sown in blocks of a single genotype. The GUA is an important factor in determining the nature of a host-diversity effect on disease (50). The ideal GUA for reduced disease may be infinitely small, so genotypes are perfectly mixed, although unusual combinations of genotype patterning and dispersal gradients may result in other GUA optima. Inherent differences in GUA between host species may lead to different magnitudes of host-diversity effects. Genotype mixtures of large crop plants have sometimes demonstrated smaller hostdiversity effects than those typically observed in small-grain mixtures $(12,31,49,50,56)$. In contrast, host-diversity effects comparable to those in mixtures of small-grain plants have been noted, in some cases, in large plants for both Phytophthora infestans on potato (K. A. Garrett and C. C. Mundt, unpublished data) and Melampsora epitea var. epitea on willow (36). The impact of GUA has been studied experimentally by altering the degree of aggregation of plants of the same genotype. Increasing GUA through higher aggregation usually has decreased host-diversity effects for reduced disease $(31,50,51)$. For the range of GUA considered, GUA was more important as a determinant for host-diversity effects on corn rust than on bean rust (50). On the other hand, within-hill versus between-hill mixtures of rice genotypes did not result in a greatly different effect for reduced blast caused by Magnaporthe grisea (summarized in Mundt [40]). The importance of GUA for hostdiversity effects can be strongly influenced by the spatial pattern of initial disease. In studies of Puccinia coronata on oat, mixtures with large GUAs produced a larger decrease in disease when the disease was focal $(45,48)$, i.e., disease spread from a small number of locations rather than being more evenly spread throughout host plants at the beginning of an epidemic (48). These results have been corroborated through simulation modeling (49).

\section{Lesion Expansion and Limits}

\section{to the Host's Carrying Capacity for Disease}

If individual lesions can expand, such expansion also decreases any disease reduction due to host diversity. Infection of new
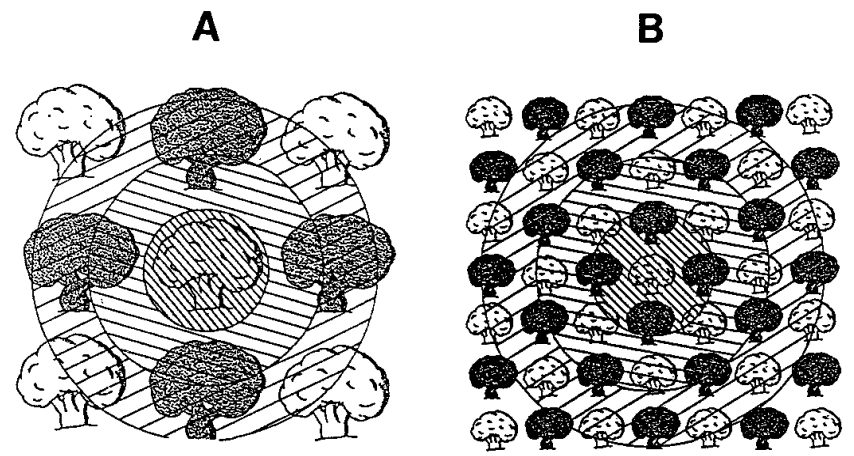

Fig. 1. Pathogen dispersal gradient superimposed over two plant populations. Within a population, two different host genotypes are indicated by different shading of individual plants. Concentric circles indicate pathogen propagule dispersal originating from the center host plant, with propagule loads decreasing with distance from the source. A, When the size of individual plants is large relative to the dispersal gradient, inoculum may be concentrated on the host plant where the inoculum originates. B, When the size of individual plants is small relative to the dispersal gradient, a greater share of inoculum may fall on plants with a genotype different from the source host plant. 
tissues due to expansion will automatically occur on susceptible plants, an effect similar to autoinfection. In the past, lesion expansion was given relatively little consideration as an epidemic component, but recent work suggests that lesion expansion may be a critical determinant of epidemic progression in many pathosystems (7). Lannou et al. (32,33) studied the influence of lesion expansion on host-diversity effects in a computer simulation model and found that lesion expansion could substantially decrease hostdiversity effects for reduced disease. They also compared wheat stripe rust (caused by Puccinia striiformis), with substantial lesion expansion, and wheat leaf rust (caused by Puccinia recondita), with determinant lesions, in empirical studies. When environment and pathogen generation time were held constant, their results suggested that lesion expansion of stripe rust decreased host-diversity effects for reduced disease by half compared with leaf rust (32).

Equation 1 does not account for the effect of the limits to a host's carrying capacity for disease. Because host-diversity effects accumulate over generations, they are greatly reduced if disease progress follows an asymptotic form, such as a logistic growth model, rather than increasing indefinitely. This may explain some cases in which host-diversity effects have been smaller in the field than predicted by the classic model (39). In addition, the effect of latent infection on the host's carrying capacity for disease (21) may decrease the host-diversity effect for reduced disease further (41). In nature, conditions are rarely continuously conducive to increasing disease. An epidemic interrupted frequently by periods of weather unfavorable to disease increase may result in a host-diversity effect close to that predicted by a logarithmic growth model. Thus, in the field, host-diversity effects likely will fall somewhere along the continuum between the effects predicted by a logarithmic and those predicted by a logistic growth model.

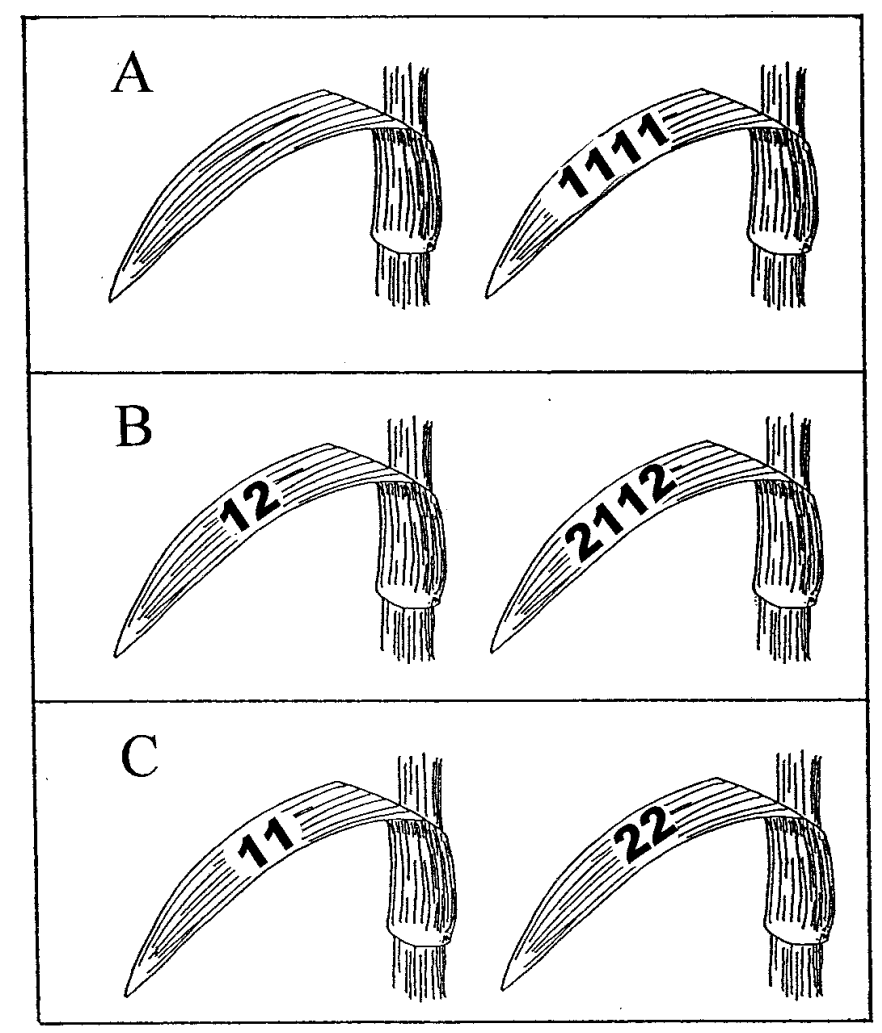

Fig. 2. Leaves in three types of genotype mixtures. For each mixture, one leaf from each of two genotypes is represented. Numerals 1 and 2 on leaves represent individual lesions from races 1 and 2 of a pathogen, respectively. A, For simplest mixtures, only one genotype is susceptible. B, For genotypes with different levels of race-nonspecific resistance, both genotypes may be infected by the same races but with different levels of severity. $\mathbf{C}$, For genotypes with different levels of race-specific resistance (differential susceptibility), genotypes may be infected with different races.

\section{Spatial Extent of Mixture Incidence}

We considered the scale, or spatial grain size, of genotypic variation in a mixture in terms of GUA. The extent of mixture plantings is also a factor. Use of mixtures over a larger area may increase the host-diversity effect for reduced disease, even when the GUA is large (42). In the former East Germany, both the severity of barley powdery mildew infection and fungicide use dropped substantially over time as the total barley area planted to mixtures rose from $0 \%$ during the early 1980 s to $92 \%$ of the total barley area planted in 1990 (64). Similar, but much less extensive, observations of increased host-diversity effects at larger spatial scales have been made for wheat stripe rust in the Pacific Northwest of the United States (40). Mixtures of coffee genotypes have been planted on a large scale in an effort to proactively reduce anticipated infection by Hemileia vastatrix in Colombia (38), although the epidemiological impact of this strategy has yet to be reported. Modeling studies (42) suggest that the number of genotype units may be more important than GUA per se. This hypothesis is extremely difficult to test experimentally, but one attempt suggests the impact of the number of genotype units may or may not be detected, depending on the wind characteristics of the particular site and spatial pattern of the units (43).

Based on a theory of linear expansion of disease foci, Van den Bosch et al. (60) developed a model that predicts the velocity of focus expansion in simplest mixtures as increasing linearly with the logarithm of the proportion of susceptible plants in the mixture. This logarithmic relationship has been corroborated through empirical studies in small field plots for rust diseases of both wheat (60) and bean (4). However, for wind-dispersed pathogens whose propagules escape from the canopy, the velocity of focus expansion may increase with distance from the inoculum source (15). If this is the case, then a host-diversity effect for reduced disease may be larger for larger fields of mixtures than for smaller fields, because the difference in the velocity of the epidemic between pure and mixed stands would increase with distance from the inoculum source.

Other procedures commonly used in small-scale field experiments may result in greatly underestimated host-diversity effects for reduced disease in larger populations or commercial agricultural production. Experiments comparing mixtures and single-genotype populations have been postulated to be highly sensitive to the effects of interplot interference $(40,63)$. Reduced infection efficiency, as described in equation 1 , is generally the primary mechanism by which mixtures may reduce disease severity, but interplot interference due to spore dispersal from single-genotype susceptible host plots effectively increases the infection efficiency of the pathogen population in a mixture (40). In contrast, increased latent periods associated with horizontal resistance, for example, would not be directly influenced by interplot interference. Further, although host-diversity effects for reduced disease increase with generation number (equation 1), interplot interference and artificial inoculation in small-scale field experiments can reduce the total number of generations of pathogen increase required for epidemic completion. A recent experiment in China showed a 92 to $95 \%$ reduction of rice blast severity due to mixing in a large-scale experiment incorporating 812 ha and designed with controls that minimized interplot interference (69). In contrast, a literature review of smallplot studies indicated a mean disease reduction of only $50 \%$ (40).

\section{GENETIC REFINEMENTS TO THE CLASSIC MODEL}

In simplest mixtures, one component is immune while the other is susceptible (Fig. 2A). This is often true of mixtures of different plant species and occasionally true of mixtures of host genotypes within a species. For some host species, genotypes with immunity may not exist. In this case, all host genotypes express some degree of susceptibility (Fig. 2B). Host genotypes may have race-specific differences in resistance, so they are differentially susceptible to 
local pathogen races. For such mixtures, some pathogen races will tend to infect certain host genotypes, while other races will tend to infect different host genotypes (Fig. 2C). For the moment, we will disregard the effects of spatial pattern.

\section{Race-Nonspecific Differences in Resistance of Host Genotypes}

Host mixtures may be composed of genotypes with varying levels of race-nonspecific resistance with respect to the local pathogen population (Fig. 2B). In this case, the nature of the host-diversity effect on disease will depend, to a large degree, on whether the disease decrease on the more susceptible cultivar is greater than the disease increase on the more resistant cultivar. The reduction in susceptible host tissue becomes a more complicated issue in this scenario. The more susceptible genotype will experience a decreased inoculum load in contrast to being grown as a single genotype, but the resistant genotype will experience an increased load. Variable effects of host diversity have been reported for such mixtures (1, $25,37,46,53)$.

Jeger et al. (24) modeled the influence of differences in racenonspecific resistance on mixtures by assuming spatial homogeneity of host and pathogen as in Leonard's model (35). Their model assumes nonspecificity in the sense that the relative resistance of the host genotypes is constant for each genotype of the pathogen population. In the model, resistance is split into two components: infection frequency and sporulation rate. A host-diversity effect for increased disease is predicted when one host genotype has a greater infection frequency and the other has a greater sporulation rate. This combination is unusual; the more common scenario would be for one genotype to be more resistant on both counts, leading to a host-diversity effect for reduced disease. Such a mixture would be expected to produce a host-diversity effect due to a reduced proportion of susceptible tissue similar to that predicted by Leonard (35); although rather than $m^{n}$, the multiplier for the proportion of diseased leaf tissue in monoculture would be approximately $[m+$ $(1-m) z]^{n}$, where $z$ is the ratio of the susceptibility of the resistant genotype to that of the susceptible genotype. For example, if the more resistant genotype is completely immune, the ratio $z$ is 0 , and Leonard's case (35) is given. If the more resistant genotype is only slightly more resistant than the susceptible genotype, ratio $z$ will be near 1 , and there will be almost no host-diversity effect due to a reduction in susceptible host tissue.

In most cases, the differences between host genotypes will not be completely race nonspecific, and true nonspecificity may be impossible to predict (26). However, a model such as this can approximate results when race specificity is weak if the spatial aspects of the system do not override it.

\section{Race-Specific Differences in Resistance of Host Genotypes}

If populations of individuals within pathogenic races quantitatively adapt to different host genetic backgrounds in mixtures, this may cause disruptive selection to races that are able to attack more than one host genotype in a mixture. For barley powdery mildew, differences in host genetic background were estimated to account for $\approx 25 \%$ of the total host-diversity effect (67), and disruptive selection reduced the fitness of a complex race (13). In a mixture of a susceptible and moderately resistant wheat genotype, epidemics caused by Septoria tritici were suppressed to the level of the moderately resistant genotype as the season progressed (47). Quantitative adaptation of the pathogen to the genetic background of the two genotypes, which previously had been demonstrated in the pathosystem, probably played a role in suppression.

Host-diversity effects for decreased disease can be increased dramatically if the host genotypes express differential qualitative resistance to races of the pathogen. Such mixtures function similarly to the simplest mixtures considered by Leonard (35), except that each component is the resistant component for a subset of local pathogen races and the susceptible component for other local races (Fig. 2C). Each host genotype has the potential to benefit from being in a mixed population because of the reduced proportion of tissue that is susceptible to races that can infect it. Even if differentially susceptible mixtures have higher overall disease levels than simplest mixtures, there is the potential for a much greater host-diversity effect on disease because disease levels on all genotypes may be affected.

Additional mechanisms for host-diversity effects may come into play for mixtures with differential resistance. In mixtures of an immune and susceptible genotype, the decreased proportion of susceptible tissue is probably the primary mechanism for a host-diversity effect $(9,13,63)$, with physical barriers to spread from one susceptible plant to another and the effects of compensation or competition (discussed below) also potentially playing a role. These three mechanisms all may be operative for mixtures with differential resistance. In addition, as a fourth mechanism, there is increased potential for induced host resistance in host mixtures. Resistance may be induced when propagules produced on neighbors fall on a plant for which they are avirulent. Calonnec et al. (11) estimated that one-third of the reduction in infection by Puccinia striiformis in wheat mixtures was due to induced resistance. They also concluded that induced resistance was particularly important in the system because of greater lesion expansion in its absence. With barley powdery mildew, induced resistance played a greater role during later stages of epidemic development, when higher disease severity increased the probability of interaction among races (13). A simulation study indicated that the area of tissue induced to resistance around an attempted infection is a critical factor determining the role of induced resistance in mixtures (34).

Interactions between pathogen races may form a fifth mechanism for host-diversity effects. Interactions probably will occur more frequently in host mixtures because there is likely to be a higher diversity of pathogen genotypes in host mixtures than in populations of a single host genotype. Competition among coinoculated races results in decreased disease severity in some pathosystems (55), and more competitive races are not necessarily more fit (54). Although competition between races may play an important role in determining host-diversity effects, it has not received much attention, perhaps because of the challenges of studying pathogen interactions.

For differentially susceptible mixtures, increasing the number of host genotypes can produce increased host-diversity effects. For a given GUA, similar genotypes are spaced further apart as the number of genotypes increases. Greater host-diversity effects for reduced disease have been observed as the number of host genotypes increases for Rhynchosporium secalis on barley (52) and for Puccinia striiformis on wheat (40).

\section{Compensation and Competition}

Unless the genotypes that make up a host mixture are isolines, other aspects of their genetic background are likely to influence host-diversity effects on disease. Growth compensation by resistant plant genotypes can be important, especially when there are innate competitive differences among host genotypes that are exaggerated by effects of disease on plant competition and when disease occurs early in the life cycle of the host. For mixtures of wheat genotypes infected with Puccinia striiformis, Finckh and Mundt $(16,17)$ found that increased tillering of the resistant genotype sometimes accounted for very substantial proportions of the total disease reduction in mixtures. Occasionally, however, the competitive success of a susceptible genotype resulted in an increase in overall disease severity. Alexander et al. (3) also found that the more susceptible host genotype in mixtures they studied was more competitive. In a study subsequent to Finckh and Mundt $(16,17)$, Akanda and Mundt (2) used a different set of races for inoculation; as a result, some of the same host genotype mixtures used by Finckh and Mundt $(16,17)$ were differentially susceptible, i.e., the host genotypes exhibited differential race-specific resistance. In this case, different degrees of tillering had almost no impact on 
overall severity levels in the mixtures. This presumably was due to the frequency dependence of disease, which resulted in increased disease severity and reduced host fitness of the more competitive genotypes in the mixtures. Plant competition in mixtures sometimes also may alter the susceptibility of a given host genotype, altering host-diversity effects for disease (16).

Compensation may result in increased yields in mixtures even if disease is not affected, as is suggested by positive yield responses for mixtures in the presence of soilborne pathogens such as Cephalosporium gramineum on wheat (44) and Phytophthora sojae on soybean (62).

\section{PREDICTING HOST-DIVERSITY EFFECTS IN SPECIFIC HOST-PATHOGEN SYSTEMS}

An ideal theory of epidemiology in diverse host populations would allow us to accurately predict host-diversity effects for any given host-pathogen system. As discussed above, host-diversity effects on disease can be predicted in a qualitative manner by determining how a particular system deviates from the characteristics of simplest mixtures. Some deviations are inherent to a host-pathogen combination, while others are a function of environmental influences and management decisions. Inherent characteristics of systems are those that are more or less fixed for a particular system, such as plant size (and GUA) and the pathogen's dispersal gradient. Although these characteristics vary from one environment to another for specific systems, there are consistent qualitative differences in characteristics between systems. Other characteristics, such as host population size and number of host genotypes, may exhibit more variability between populations in nature and can be manipulated in agricultural systems. An ideal theory also would allow the formulation of optimal strategies for selecting plant genotypes for mixtures and deploying them in agricultural systems for disease management.

\section{Comparing the Inherent Characteristics of Host-Pathogen Systems}

We summarize the inherent differences between host-pathogen systems in terms of five major characteristics (Table 1). We would predict larger host-diversity effects for reduced disease for smaller plants, such as wheat and rice. Flatter dispersal gradients, as for Puccinia spp., would predict for larger host-diversity effects, whereas the smaller lesions of $P$. recondita would predict for a larger hostdiversity effect than the expanding lesions of $P$. striiformis. Shorter generation times predict for larger host-diversity effects, favoring effects for diseases such as mildews. Finally, greater specialization of pathogen populations would be predicted to lead to greater host-diversity effects, especially for differentially susceptible mixtures. Determining whether published empirical studies validate these predictions is complicated by three factors. First, the number of systems in which mixtures have been studied makes up only a small percentage of those addressed by plant pathologists, and although $P$. recondita and $P$. striiformis have been compared directly as a test of the influence of lesion size (32), experiments directly comparing host-diversity effects for different diseases are rare. Second, some of the inherent characteristics of specific host-pathogen systems may predict for a host-diversity effect, whereas other characteristics of the same system do not. How to weight the different factors in Table 1 and take into account their interactions are open questions. Some hosts with larger GUAs, such as pepper and potato, have demonstrated host-diversity effects for reduced infection by Xanthomonas campestris pv. vesicatoria (31) and Phytophthora infestans (D. Andrivon, unpublished data; K. A. Garrett and C. C. Mundt, unpublished data), respectively. Phytophthora infestans and $X$. campestris pv. vesicatoria, which produce expanding lesions in addition to parasitizing large hosts, might be predicted to experience a small host-diversity effect. Yet, their shorter generation times and the flatter dispersal gradient of $P$. infestans argue for a larger host-diversity effect (Table 1). Soilborne pathogens, such as Rhizoctonia cerealis, would not be likely candidates for host-diversity effects for reduced disease (Table 1), but Vilich-Meller (61) observed such host-diversity effects in some cases for unknown reasons. Third, comparisons between experiments are particularly complicated for mixture studies because of the strong impact of environmental differences from season to season. Environmental variability not only has a direct effect on disease development but also on competition between plant genotypes and potentially on interactions between pathogen genotypes.

\section{Influence of Environment and Management Decisions}

Among other factors, environmental variation from site to site and season to season influence the severity of epidemics. How epidemic severity influences host-diversity effects is difficult to predict using current theory. On the one hand, a faster approach to carrying capacity may decrease host-diversity effects for reduced disease $(39,49)$. On the other hand, if a severe epidemic is caused primarily by an earlier onset of disease and, thus, a large number of pathogen generations, it may increase host-diversity effects for reduced disease (equation 1). Further, the severity of epidemics may influence the relative importance of outside inoculum, with outside inoculum making up a larger share of the total inoculum in a host population when epidemics are less severe.

Management comes into play in agricultural systems through the number of plant genotypes included and planting density. At this point, it seems clear that host-diversity effects for reduced disease usually will be larger for mixtures constructed of either many genotypes (if differentially susceptible) or a small proportion of susceptible plants. The fitness of host genotypes may be frequency dependent, with or without disease (18). Planting density may influence host-diversity effects, both through a modified microclimate and its influence on host GUA. Higher host density may result in more rapid epidemics, but density can be challenging to manipulate

TABLE 1. Inherent characteristics that predict host-diversity effect for reduced disease and whether illustrative host-pathogen systems possess these characteristics

\begin{tabular}{|c|c|c|c|c|c|c|}
\hline \multirow[b]{2}{*}{ Host } & \multirow[b]{2}{*}{ Pathogen } & \multicolumn{5}{|c|}{ Characteristic $^{\mathrm{a}}$} \\
\hline & & $\begin{array}{c}\text { Small host } \\
\text { genotype unit area }\end{array}$ & $\begin{array}{c}\text { Shallow dispersal } \\
\text { gradient }\end{array}$ & Small lesion size & $\begin{array}{l}\text { Short pathogen } \\
\text { generation time }\end{array}$ & $\begin{array}{c}\text { Strong host } \\
\text { specialization }^{\mathrm{b}}\end{array}$ \\
\hline Coffee & Hemileia vastatrix & - & + & + & - & + \\
\hline Potato & Phytophthora infestans & - & + & - & + & + \\
\hline Rice & Magnaporthe grisea & + & + & + & + & + \\
\hline \multirow[t]{3}{*}{ Wheat } & Erysiphe graminis f. sp. tritici & + & + & + & + & + \\
\hline & Puccinia recondita & + & + & + & - & + \\
\hline & Rhizoctonia cerealis & + & - & - & - & - \\
\hline
\end{tabular}

\footnotetext{
a -, Host-pathogen system does not have the characteristic, so a host-diversity effect for reduced disease is less likely; +, host-pathogen system has the characteristic,
} so a host-diversity effect for reduced disease is more likely.

${ }^{\mathrm{b}}$ High degree of host specialization in local pathogen populations. 
in host species that can compensate well for planting rate (57). Increased planting density may result in greater disease reductions due to mixing if plant competition causes a reduced GUA (6), but recent work with Puccinia striiformis on wheat suggests that hostdiversity effects may be greatest at intermediate densities (K. A. Garrett and C. C. Mundt, unpublished data). Although most studies of plant diseases have involved agricultural systems, interest in disease is growing among plant ecologists. Knops et al. (28) observed that disease severity decreased with increasing species diversity within experimental plots of grassland plants.

To test whether mixtures are useful in deployment of disease resistance for a particular host-pathogen system, simple mixtures of susceptible and very resistant genotypes may be used to determine whether the three basic mechanisms, in particular dilution of susceptible host tissues, are active for that system. If the GUA is very large and the dispersal gradient is very steep, there may be little or no host-diversity effect. If simple mixtures reveal a host-diversity effect for reduced disease, the next step might be to work with differential resistance, if useful, as well as adding high levels of horizontal resistance.

A basic decision in agricultural systems is whether to use crop plant mixtures. Although genotype and species mixtures are common in traditional agriculture, there is less awareness of the increased use of genotype mixtures in commercial agriculture. For example, $92 \%$ of the more than 300,000 ha of barley grown in the former East Germany were planted to genotype mixtures before reunification (65). Genotype and species mixtures also are gaining significantly in popularity in several European countries (66), while wheat genotype mixtures are increasing in popularity in the $\mathrm{Pa}$ cific Northwest of the United States (40). In 1998, $10 \%$ of the soft white winter wheat production area of Oregon was sown to genotype mixtures, for a total of 32,000 ha (30). In Washington State in $1998,12.7 \%$ of the soft white winter wheat production area and $76 \%$ of the club wheat production area were sown to genotype mixtures, for a total of 158,000 ha (23). Wheat genotype mixtures also have been investigated in Kansas, with positive grower response (27). During the 1997 to 1998 and 1998 to 1999 winter wheat seasons, 2.6 and $6.1 \%$ of the Kansas wheat crops, respectively, were sown to cultivar mixtures, for a total of 106,000 and 227,000 ha, respectively (W. W. Bockus, personal communication).

Several potential benefits of using crop genotype mixtures may be responsible for their increased use in commercial agriculture. As noted in the introduction, one motive may be to decrease selection pressures for pathogen genotypes able to overcome particular host genes for resistance. From a short-run perspective, mixtures may be useful for disease management in host-pathogen systems for which there is a host-diversity effect for reduced disease. First, suppose immunity or very strong resistance is available in one subset of plant genotypes, but other genotypes are more valuable, more desirable agronomically, or have seed that is less expensive. In this case, a mixture with a genetic composition similar to simplest mixtures may be advantageous. A more common scenario, however, is that plant genotypes with differential resistance are available. A mixture of these genotypes may be advantageous compared with growing any one of them alone, because the pathogen population would be partitioned between different genotypes. In addition to disease management, common reasons for agricultural use of mixtures are to reduce the risk of yield loss caused by other biotic and abiotic stresses, to reduce yield variability caused by cultivarenvironment interactions, and to take advantage of yield compensation occurring in mixtures grown in variable environments (C. C. Mundt, unpublished data).

Plant genotypes selected for agricultural mixtures should be chosen to exhibit complementary growth traits, as well as complementary resistance characteristics. Genotypes could be tested in mixing ability analyses, similar to combining ability analyses in plant breeding, to learn which genotypes create mixtures that are better for disease control and yield (29). In differential mixtures, Akanda and Mundt (2) found that the host-diversity effect on disease varied for a given host genotype, depending on which differentially resistant genotype was included with it in a two-component mixture. When information about local pathogen populations is available, differentially susceptible mixtures may be tailored to maximize resistance against the population (68). Customizing mixtures to local pathogen populations will be an important next step in the application of mixture theory for disease management.

\section{ACKNOWLEDGMENTS}

This work was supported in part by USDA NRICGP 96-35303-3206 and by US-AID funding for CGIAR-University Linkage. We thank C. Cowger, D. J. Coyle, M. E. Daub, A. M. Jarosz, R. J. Nelson, W. F. Pfender, and an anonymous reviewer for comments that improved this manuscript. We thank K. Merrifield for the illustrations.

\section{LITERATURE CITED}

1. Ahmed, H. U., Finckh, M. R., Alfonso, R. F., and Mundt, C. C. 1997. Epidemiological effect of gene deployment strategies on bacterial blight of rice. Phytopathology 87:66-70.

2. Akanda, S. I., and Mundt, C. C. 1996. Effects of two-component wheat cultivar mixtures on stripe rust severity. Phytopathology 86:347-353.

3. Alexander, H. M., Roelfs, A. P., and Cobbs, G. 1986. Effects of disease and plant competition on yield in monocultures and mixtures of two wheat cultivars. Plant Pathol. 35:457-465.

4. Assefa, H., van den Bosch, F., and Zadoks, J. C. 1995. Focus expansion of bean rust in cultivar mixtures. Plant Pathol. 44:503-509.

5. Barrett, J. A. 1980. Pathogen evolution in multilines and variety mixtures. J. Plant Dis. Prot. 87:383-396.

6. Barrett, J. A., and Wolfe, M. S. 1980. Pathogen response to host resistance and its implication in breeding programmes. Eur. Mediterr. Plant Prot. Org. Bull. 10:341-347.

7. Berger, R. D. 1997. Lesion expansion as an epidemic component. Phytopathology 87:1005-1013.

8. Burdon, J. J. 1987. Disease and Plant Population Biology. Cambridge University Press, Cambridge.

9. Burdon, J. J., and Chilvers, G. A. 1977. Controlled environment experiments on epidemic rates of barley mildew in different mixtures of barley and wheat. Oecologia 28:141-146.

10. Burdon, J. J., Jarosz, A. M., and Kirby, G. C. 1989. Pattern and patchiness in plant-pathogen interactions - Causes and consequences. Annu. Rev. Ecol. Syst. 20:119-136.

11. Calonnec, A., Goyeau, H., and de Vallavieille-Pope, C. 1996. Effects of induced resistance on infection efficiency and sporulation of Puccinia striiformis on seedlings in varietal mixtures and on field epidemics in pure stands. Eur. J. Plant Pathol. 102:733-741.

12. Chakraborty, S., Pettitt, A. N., Cameron, D. F., Irwin, J. A. G., and Davis, R. D. 1991. Anthracnose development in pure and mixed stands of the pasture legume Stylosanthes scabra. Phytopathology 81:788-793.

13. Chin, K. M., and M. S. Wolfe. 1984. The spread of Erysiphe graminis f. sp. hordei in mixtures of barley varieties. Plant Pathol. 33:89-100.

14. Dwyer, G., Elkinton, J. S., and Buonaccorsi, J. P. 1997. Host heterogeneity in susceptibility and disease dynamics: Tests of a mathematical model. Am. Nat. 150:685-707.

15. Ferrandino, F. J. 1993. Dispersive epidemic waves: I. Focus expansion within a linear planting. Phytopathology 83:795-802.

16. Finckh, M. R., and Mundt, C. C. 1992. Plant competition and disease in genetically diverse wheat populations. Oecologia 91:82-92.

17. Finckh, M. R., and Mundt, C. C. 1992. Stripe rust, yield, and plant competition in wheat cultivar mixtures. Phytopathology 82:905-913.

18. Finckh, M. R., and Mundt, C. C. 1993. Effects of stripe rust on the evolution of genetically diverse wheat populations. Theor. Appl. Genet. 85: 809-821.

19. Fitt, B. D. L., Gregory, P. H., Todd, A. D., McCartney, H. A., and MacDonald, O. C. 1987. Spore dispersal and plant disease gradients: A comparison between two epidemic models. J. Phytopathol. 118:227-242.

20. Fitt, B. D. L., and McCartney, H. A. 1986. Spore dispersal in relation to epidemic models. Pages 311-345 in: Plant Disease Epidemiology, vol. 1. K. J. Leonard and W. E. Fry, eds. Macmillan Publishing Company, New York.

21. Fleming, R. A. 1983. Development of a simple mechanistic model of cereal rust progress. Phytopathology 73:308-312.

22. Goliniewski, G., and Newton, A. C. 1994. Modelling the spread of fungal diseases using a nearest neighbour approach: Effect of geometrical arrangement. Plant Pathol. 43:631-643.

23. Hasslen, D., and McCall, J. 1998. 1998 Annual Bulletin—Wheat. Published 
on-line by the USDA National Agricultural Statistics Service/Washington Agricultural Statistics Service. On-line publication annual98/wheat 98 .

24. Jeger, M. J., Griffiths, E., and Jones, D. G. 1981. Disease progress of nonspecialized fungal pathogens in intraspecific mixed stands of cereal cultivars. I. Models. Ann. Appl. Biol. 98:198.

25. Jeger, M. J., Jones, D. G., and Griffiths, E. 1981. Disease progress of nonspecialised fungal pathogens in intraspecific mixed stands of cereal cultivars. II. Field experiments. Ann. Appl. Biol. 98:199-210.

26. Johnson, R. 1981. Durable resistance: Definition of, genetic control and attainment in plant breeding. Phytopathology 81:567-568.

27. Kessler, K. 1997. Wheat blends stabilize yields. Furrow, Jan., pp. 27-28.

28. Knops, J. M. H., Tilman, D., Haddad, N. M., Naeem, S., Mitchell, C. E., Haarstad, J., Ritchie, M. E., Howe, K. M., Reich, P. B., Siemann, E., and Groth, J. Effects of plant species richness on invasions dynamics, disease outbreaks, insects abundances, and diversity. Ecol. Lett. 2. In press.

29. Knott, E. A., and Mundt, C. C. 1990. Mixing ability analysis of wheat cultivar mixtures under diseased and nondiseased conditions. Theor. Appl. Genet. 80:313-320.

30. Korn, V. G., ed. 1998. 1997-1998 Oregon Agriculture and Fisheries Statistics. USDA Nature and Agricultural Station Service and Oregon Department of Agriculture, Salem, OR.

31. Kousik, C. S., Sanders, D. C., and Ritchie, D. F. 1996. Mixed genotypes combined with copper sprays to manage bacterial spot of bell peppers. Phytopathology 86:502-508.

32. Lannou, C., de Vallavieille-Pope, C., Biass, C., and Goyeau, H. 1994. The efficacy of mixtures of susceptible and resistant hosts to two wheat rusts of different lesion size: Controlled condition experiments and computerized simulations. J. Phytopathol. 140:227-237.

33. Lannou, C., de Vallavieille-Pope, C., and Goyeau, H. 1994. Host mixture efficacy in disease control: Effects of lesion growth analyzed through computer-simulated epidemics. Plant Pathol. 43:651-662.

34. Lannou, C., de Vallavieille-Pope, C., and Goyeau, H. 1995. Induced resistance in host mixtures and its effect on disease control in computersimulated epidemics. Plant Pathol. 44:478-489.

35. Leonard, K. J. 1969. Factors affecting rates of stem rust increase in mixed plantings of susceptible and resistant oat varieties. Phytopathology 59: 1845-1850.

36. McCracken, A. R., and Dawson, W. M. 1998. Short rotation coppice willow in Northern Ireland since 1973: Development of the use of mixtures in the control of foliar rust (Melampsora spp.). Eur. J. For. Pathol. 241-250.

37. McDonald, B. A., Allard, R. W., and Webster, R. K. 1988. Responses of two-, three-, and four-component barley mixtures to a variable pathogen population. Crop Sci. 28:447-452.

38. Moreno Ruiz, G., and Castillo Zapata, J. 1990. The Variety Colombia: A Variety of Coffee with Resistance to Rust (Hemileia vastatrix Berk. \& Br.), CENICAFE, Chinchina-Caldas, Colombia.

39. Mundt, C. C. 1990. Disease dynamics in agroecosystems. Pages 263-299 in: Agroecology. C. R. Carroll, J. H. Vandermeer, and P. M. Rossett, eds. McGraw-Hill Publishing Co., New York.

40. Mundt, C. C. 1994. Use of host genetic diversity to control cereal diseases: Implications for rice blast. Pages 293-308 in: Rice Blast Disease. R. S. Zeigler, S. A. Leong, and P. S. Teng, eds. CAB International, Wallingford, England, UK.

41. Mundt, C. C. 1997. Disease increase in host mixtures. Pages 119-122 in: Exercises in Plant Disease Epidemiology. L. J. Francl and D. A. Nehr, eds. The American Phytopathological Society, St. Paul, MN.

42. Mundt, C. C., and Brophy, L. S. 1988. Influence of number of host genotype units on the effectiveness of host mixtures for disease control: A modeling approach. Phytopathology 78:1087-1094.

43. Mundt, C. C., and Brophy, L. S., and Kolar, S. C. 1996. Effect of genotype unit number and spatial arrangement on severity of yellow rust in wheat cultivar mixtures. Plant Pathol. 45:215-222.

44. Mundt, C. C., Brophy, L. S., and Schmitt, M. E. 1995. Disease severity and yield of pure-line wheat cultivars and mixtures in the presence of eyespot, yellow rust, and their combination. Plant Pathol. 44:173-182.

45. Mundt, C. C., and Browning, J. A. 1985. Development of crown rust epidemics in genetically diverse oat populations: Effect of genotype unit area. Phytopathology 75:607-610.

46. Mundt, C. C., Hayes, P. M., and Schon, C. C. 1994. Influence of barley variety mixtures on severity of scald and net blotch and on yield. Plant Pathol. 43:356-361.

47. Mundt, C. C., Hoffer, M. E., Ahmed, H. U., Coakley, S. M., DiLeone, J. A., and Cowger, C. 1999. Population genetics and host resistance. Pages 115130 in: Septoria on Cereals: A Study of Pathosystems. J. A. Lucas, P. Bowyer, and H. M. Anderson, eds. CAB International, Wallingford, England, UK.

48. Mundt, C. C., and Leonard, K. J. 1985. Effect of host genotype unit area on epidemic development of crown rust following focal and general inoculations of mixtures of immune and susceptible oat plants. Phytopathology 75:1141-1145.

49. Mundt, C. C., and Leonard, K. J. 1986. Analysis of factors affecting disease increase and spread in mixtures of immune and susceptible plants in computer-simulated epidemics. Phytopathology 76:832-840.

50. Mundt, C. C., and Leonard, K. J. 1986. Effect of host genotype unit area on development of focal epidemics of bean rust and common maize rust in mixtures of resistance and susceptible plants. Phytopathology 76:895900.

51. Mundt, C. C., Leonard, K. J., Thal, W. M., and Fulton, J. H. 1986. Computerized simulation of crown rust epidemics in mixtures of immune and susceptible oat plants with different genotype unit areas and spatial distributions of initial disease. Phytopathology 76:590-598.

52. Newton, A. C., Ellis, R. P., Hackett, C. A., and Guy, D. C. 1997. The effect of component number on Rhynchosporium secalis infection and yield in mixtures of winter barley cultivars. Plant Pathol. 45:930-938.

53. Newton, A. C., and Thomas, W. T. B. 1992. The effect of specific and nonspecific resistance in mixtures of barley or genotypes on infection by mildew (Erysiphe graminis f. sp. hordei) and on yield. Euphytica 59:73-81.

54. Newton, M. R., Kinkel, L. L., and Leonard, K. J. 1997. Competition and density-dependent fitness in a plant parasitic fungus. Ecology 78:1774-1784.

55. Newton, M. R., Kinkel, L. L., and Leonard, K. J. 1998. Determinants of density- and frequency-dependent fitness in competing plant pathogens. Phytopathology 88:45-51.

56. Ntahimpera, N., Dillard, H. R., Cobb, A. C., and Seem, R. C. 1996. Anthracnose development in mixtures of resistant and susceptible dry bean cultivars. Phytopathology 86:668-673.

57. Pfleeger, T. G., and Mundt, C. C. 1998 . Wheat leaf rust severity as affected by plant density and species proportion in simple communities of wheat and wild oats. Phytopathology 88:708-714.

58. Power, A. G. 1991. Virus spread and vector dynamics in genetically diverse plant populations. Ecology 72:232-241.

59. Robinson, R. A. 1976. Plant Pathosystems. Springer-Verlag, New York.

60. van den Bosch, F., Verhaar, M. A., Buiel, A. A. M., Hoogkamer, W., and Zadoks, J. C. 1990. Focus expansion in plant disease. IV. Expansion rates in mixtures of resistant and susceptible hosts. Phytopathology 80:598-602.

61. Vilich-Meller, V. 1992. Pseudocercosporella herpotrichoides, Fusarium spp. and Rhizoctonia cerealis stem rot in pure stands and interspecific mixtures of cereals. Crop Prot. 11:45-50.

62. Wilcox, J. R., and St. Martin. S. K. 1998. Soybean genotypes resistant to Phytophthora sojae and compensation for yield losses of susceptible isolines. Plant Dis. 82:303-306.

63. Wolfe, M. S. 1985. The current status and prospects of multiline cultivars and variety mixtures for disease resistance. Annu. Rev. Phytopathol. 23: 251-273.

64. Wolfe, M. S. 1991. Barley diseases: Maintaining the value of our varieties. Pages 1055-1067 in: Barley Genetics VI. L. Munck, ed. Munksgaard International Publishers, Copenhagen.

65. Wolfe, M. S. 1992. Maintaining the value of our varieties. Pages 1055-1067 in: Barley Genetics VI. Munksgaard International publishers, Copenhagen.

66. Wolfe, M. S. 1997. Variety Mixtures in Theory and Practice. COST Action 817. INRA, Grigon, France.

67. Wolfe, M. S., Barrett, J. A., and Jenkins, J. E. E. 1981. The use of cultivar mixtures for disease control. Pages 73-80 in: Strategies for the Control of Cereal Disease. J. F. Jenkyn and R. T. Plumb, eds. Blackwell Scientific Publications, Oxford.

68. Yong, L., and Zadoks, J. C. 1992. A decision model for variety mixtures to control yellow rust on winter wheat. Agric. Syst. 38:17-33.

69. Youyong, Z. 1999. Yunnan Lighthouse Site, China. Pages 7-23 in: RETA 5711 Exploiting Biodiversity for Sustainable Rice Pest Management. International Rice Research Institute, Manila, Philippines. 